\title{
Analysis of Arab Israeli's Sense of National Belonging Based on K-Means Cluster Algorithm
}

\author{
Wenjia Liu ${ }^{1, *}$ \\ ${ }^{1}$ School of Middle Eastern Study, Beijing International Studies University, Beijing 100024, China \\ *Corresponding author. Email: liuwenjia126@126.com
}

\begin{abstract}
The sense of national belonging is an important feature of national identity and the core factor of the establishment of a nation state.Based on the cluster analysis method in social statistics, a quantitative analysis on the sense of belonging of Arab-Israelis is conducted in this paper.First of all, the Arab-Israelis can be is divided into two groups according to age structure and educational background.The new generation of ArabIsraelis is about 30 years old and has a good education background. The older ones are 50 years old on average and with a poor education less than 10 years. Secondly, the state of national belonging of ArabIsraelis is analyzed. The results show that the state belonging of Arab-Israelis is generally good, but there is a significant difference between the old and the new generations. The sense of national belonging of the new generation of Arab-Israelis is significantly higher than that of the older generation.
\end{abstract}

Keywords: national belonging, K-means cluster analysis, Arabs, Israel

\section{基于K-means聚类算法的阿拉伯裔以色列人 国家归属感分析}

\author{
柳文佳 ${ }^{1 *}$
}

${ }^{1}$ 北京第二外国语学院, 中东学院, 中国北京, 100024

“通信作者. Email: liuwenjia126@126.com

\section{摘要}

国家归属感是国家认同的重要表现形式，是一个民族国家得以成立的核心因素。本文基于国际社会调 查项目国家归属感调查数据，采用K-means聚类分析方法，对阿拉伯裔以色列人的国家归属感进行定 量分析。首先，从年龄结构上对阿拉伯裔进行划分，新一代以色列阿拉伯人年龄在 30 岁左右，有着良 好的教育背景; 而老一代阿拉伯人年龄在平均值在 50 岁，受教育程度一般，其平均受教育年限不到 10 年。其次，分析了以色列裔阿拉伯人国家归属感情况，结果显示：阿拉伯裔以色列人的国家归属感总 体状况良好，但新老两代阿拉伯裔以色列人的国家归属程度存在明显差异。新一代阿拉伯裔以色列人 的国家归属感明显高于老一代阿拉伯裔以色列人。

关键字：国家归属感; K-means 聚类分析; 阿拉伯; 以色列

\section{1. 引言}

归属感是佛洛姆氏理论中的术语, 意指心理上的 安全感与落实感。归属感是与生俱来的, 个体作为社 会群体的一员也会有归属感, 以其文化和价值归属感 为核心 ${ }^{[1]}$ 。国家归属感指的是源于对国家的政治、经 济、文化、传统等的认可和接受, 而产生的对国家的
归属认知和感情依附。国家归属感是国家认同的重要 表现形式，是一个民族国家得以成立的核心因素。就 全球范围来看，现代民族国家的稳定与否，与其国家 内部各个族群国家认同的强度有着紧密的关联性。以 色列建国以来，阿拉伯国家和阿拉伯人与以色列的关 系一直是中东地区的核心矛盾之一，阿以关系在中东 研究中具有重要地位, 是观察和理解中东国际政治的 一个重要维度。 
访者。面对面的采访在他们的家中进行，采访用希伯 来语、阿拉伯语和俄语进行, 时长30-45分钟。调查表 主要包括关于身份、民族自豪感和对文化多样性、移 民等的态度等项目。

\section{2. 国家归属感调查问题}

2013年ISSP以色列国家认同数据集共有 368 个变量, 共为 174 名阿拉伯裔受访者, 受访者年龄分布如下图所 示。

表 1 受访者年龄分布

\begin{tabular}{lcccccc}
\hline & 个案数 & 范围 & 最小值 & 最大值 & 平均值 & 标准差 \\
\hline $\begin{array}{l}\text { 阿拉伯受 } \\
\text { 访者年龄 }\end{array}$ & 174 & 67 & 18 & 85 & 37.67 & 16.154 \\
\hline
\end{tabular}

优先聚合, 最终按昭类别的综合性质将多个品种聚合, 进而完成聚类分析的过程 ${ }^{[1]}$ 。

为揭示以色列阿拉伯人国家归属的关联性，为后 续阿拉伯人国家认同研究提供直观量化的依据, 结合 已有研究和数据分析, 本文基于聚类分析方法提出了 以色列阿拉伯人国家归属感分析模型, 以此阿拉伯人 国家归属感现状进行了定量探讨和研究。

首先, 本文基于国际社会调查项目 (International Social Survey Programme, ISSP)所提供的国家归属调 查结果数据库进行了数据篮选, 获得以色列裔阿拉伯 人国家归属调查问卷结果样本 174 条; 然后, 对数据集 进行预处理, 重点进行了评价数据属性的有效性分析 与过滤; 最后, 通过 K-means 聚类分析算法, 基于年 龄结构及教育背景对阿拉伯裔进行层次划分, 分析了 不同层面以色列裔阿拉伯人国家归属感的差异性, 为 以色列裔阿拉伯人国家认同研究奠定一定的理论基 础。.

\section{2. 样本数据集}

\subsection{ISSP简介}

国际社会调查项目 ${ }^{[3]}$ 是一个跨国合作调查项目, ISSP成立于 1984年, 是自发的非政府非盈利学术组织, 旨在推动社会科学研究的标准化和国际化, 协调跨国 跨地区的联合项目, 建立共享的社科数据库。目前会 员国已经扩大到48个国家和地区, 中国自2007年成为 会员国。该项目影响广泛, 对跨国比较研究具有重要 价值。其中ISSP在1995、2003、2013年先后三次开展 了关于国家认同的相关调查, 以色列分别在 2003 年和 2013年参加ISSP关于国家认同的调查研究, 由于以色 列社会构成的复杂性, 阿拉伯裔和犹太裔分别作为不 同的调查对象展开调查。本文采用了ISSP的2013年国 家认同模块收集的数据, 该数据集来自2013年冬季在 以色列收集的数据。采用多阶段随机抽样的方法从以 色列人口(居住在以色列至少一年)中选择 18 岁以上的受

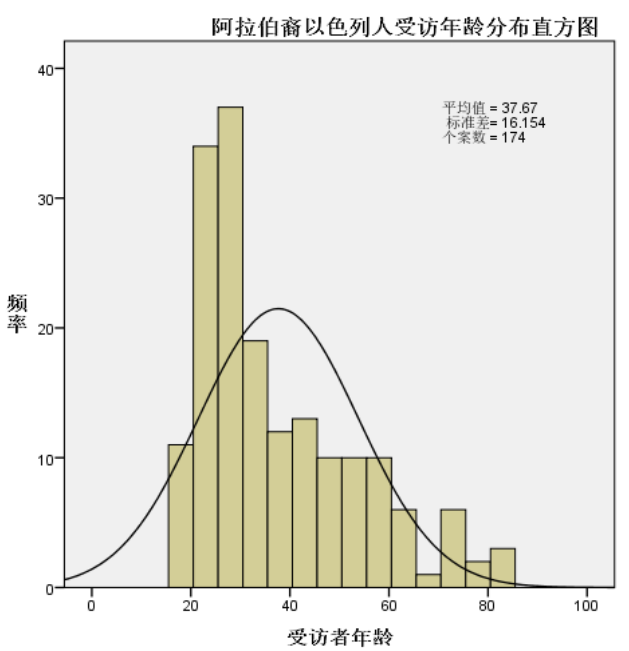

图 1 阿拉伯受访者年龄分布直方图

本文提取了其有以色列阿拉伯人涉及的国家归属 的调查的相关问题, 得出对应聚类属性: 受访者的年 龄、受教育情况以及有关国家归属的六个问题，问题 内容如下表所示。

表 2 聚类分析国家归属感涉及问题

\begin{tabular}{cr}
\hline 问题编号 & 问题内容 \\
\hline a & How important: born in [Country]? \\
b & How important: have [Country Nationality] citizenship? \\
c & How important: living most of life in [Country]? \\
d & How important: able to speak [Country Language]? \\
e & How important: to feel [Country Nationality] \\
$\mathrm{f}$ & How important: to have [Country Nationality] ancestry \\
\hline
\end{tabular}

每个问题对应选项分别为：1）非常重要；2）般重要；3）不是非常重要；4）一点也重要。 
完成, 然后生成 $0 \sim 1.0$ 之间的随机数并通过取值范围和 最小值, 以便确保随机点在数据的边界之内。

3）基于上述初始化过程，构建 K-means算法。创 建 $\mathrm{K}$ 个质心，然后将每个点分配到最近的质心，再重 新计算质心。这个过程重复数次, 直到数据点的簇分 配结果不再改变位置。返回类质心与点分配结果。停 止条件为没有需要分配的任务到不同的簇, 质心不再 发生变化, 或者均方误差 $\mathrm{E}$ 的值下降幅度最小。 $\mathrm{E}$ 的计 算公式为:

$$
E=\sum_{k=1}^{K} \sum_{x \in c_{k}} d\left(d\left(x, m_{k}\right)\right)^{2}
$$

其中, $c_{k}$ 为第 $\mathrm{k}$ 个簇; $m_{k}$ 为簇 $c_{k}$ 的质心; $d\left(x, m_{k}\right)$ 为 $\mathrm{x}$ 和质心 $m_{k}$ 之间的距离。欧氏距离是每 个目标点到簇中心的距离:

$$
\begin{aligned}
d\left(x, m_{k}\right) & =\left\|x, m_{k}\right\| \\
& =\sqrt{\left(x_{1}-m_{k}\right)^{2}+\left(x_{2}-m_{k}\right)^{2}+\ldots .+\left(x_{n}-m_{k}\right)^{2}}
\end{aligned}
$$

原始数据中, 国内出生重要性、国籍重要性、国 内居住重要性、能过说本国语言重要性、感觉到国籍 的重要性、拥有本国先祖的重要性均为属于文本类型, 不能被多因子分析方法 ${ }^{[4]}$ 直接使用。因此, 本文选用 One hot编码 ${ }^{[5]}$ 将文本数据转换成数值型, 如表 3 所示

表 3 代码属性转换表 (以 $\mathrm{k}=3$ 为例)

\begin{tabular}{|c|c|c|}
\hline 文本类型 & 表格属性 & 数值 \\
\hline & 非常重要 & 0 \\
\hline 国内出生 & 一般重要 & 1 \\
重要性 & 不是非常重要 & 2 \\
& 一点也不重要 & 3 \\
\hline
\end{tabular}

本文采用人工标定方法对样本集进行标定, 形成 标定的数据集, 来更好地评价分析模型。

\section{3. 基于K-means聚类分析算法的国家归属感 分析}

\subsection{K-means聚类分析算法}

在上述数据预处理的基础上, 将原始数据集数据 采用K-means算法进行聚类分析，其算法流程如下：

1) 将收集好的以色列阿拉伯裔国家归属特征数据 值转化为向量预先保存到文本中。

2) 构建随机质心 $O$ (中心点), 并设置 $k=2$, 即 将数据集分为“老一代以色列阿拉伯人”、“新一代以色 列阿拉伯人” 2 个簇。随机质心 $\mathrm{O}$ 在整个数据集的边界之 内, 这可以通过找到数据集每一维的最小和最大值来

\subsection{K-means聚类分析结果}

采用 K-means 聚类算法基于年龄大小，受教育年限 对受访者进行年龄结构划分，其结果如下：

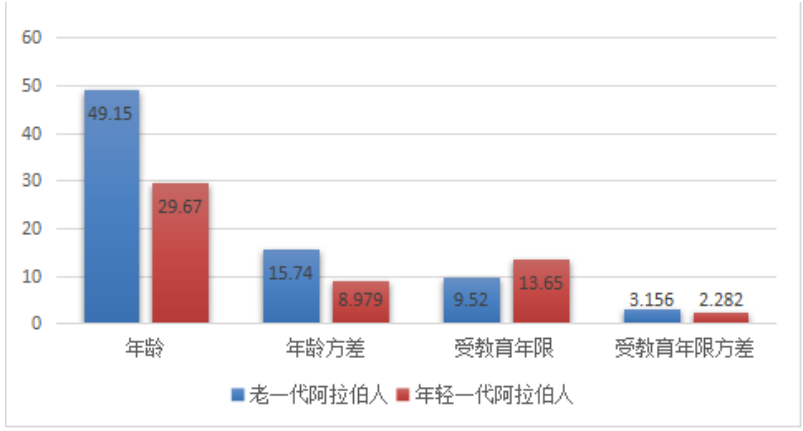

图 2 新老两代阿拉伯裔以色列人分布对比图

以受访者的年龄和受教育年限作为分类的质心， 第一类属于新一代的阿拉伯裔, 年龄在平均值在 29.67 岁, 标准差为8.979岁, 有着良好的教育背景, 其平均 受教育年限为 13.65 年; 第二类属于老一代的阿拉伯裔, 其年龄在平均值在 49.15 岁, 标准差为 15.740 岁, 受教 育程度一般，其平均受教育年限不到 10 年。

关于新老阿拉伯人对于以色列国家归属感聚类分 析据结果如下图所示: 


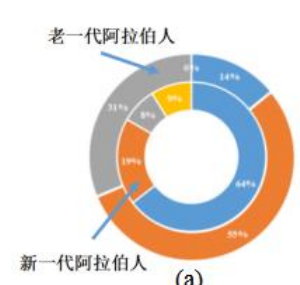

(a)

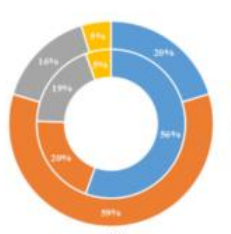

(b)

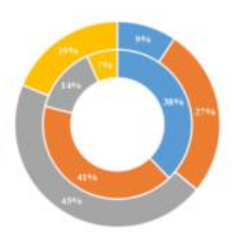

(c)

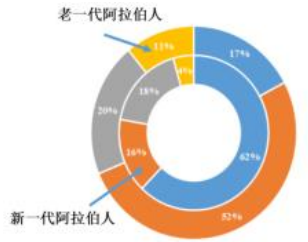

(d)

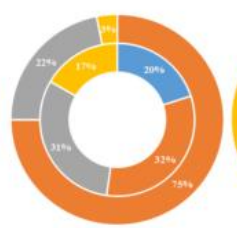

(e)

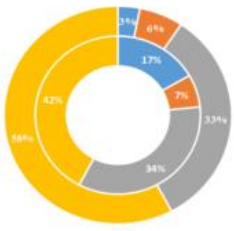

(f)

图 3 新老阿拉伯裔以色列人对社会认同的对比

可以看出, 新老阿拉伯裔以色列人在关于国家归 属感的问题的具有存在一定的差异性, 观念理解略有 不同。例如, 调查项 $\mathrm{a}$ : 在以色列出生的重要性时, 无 论是老一代阿拉伯裔以色列人还是新一代的阿拉伯都 认为在以色列出生是很重要的。但是, 具体上还有很 大的差异性，在老一代的阿拉伯裔以色列人中只有 $14 \%$ 的人认为该项非常重要, 但是在新一代阿拉伯裔以色 列人中, 这比例高达 $62 \%$ 。这是由于早期以色列对老 一代阿拉伯裔以色列人采取的政策缺乏公平性, 导致 其遭受了一定的不平等待遇。随着以色列对阿拉伯裔 以色列人的政策缓和, 新一代阿拉伯裔以色列人的国 家归属感也随之增加。对于问题c: 在以色列居住时间 的重要性是, 新老阿拉伯裔以色列人的对于此反馈的 差异性显著。在老一代阿拉伯裔以色列人中， $36 \%$ 的 受访者认为在一生的大部分时间生活在以色列是重要 的, $45 \%$ 的受访者认为其不是那么重要, 此比例占老 一代阿拉伯裔以色列人的绝大部分。而在新一代阿拉 伯裔以色列人中, $79 \%$ 的新一代阿拉伯受访者认为在 一生的大部分时间生活在以色列是重要的, 认为不那 么重要的比例只有可怜的 $14 \%$ 。这一组数字的对比充 分表现出以色列阿拉人更加倾向于在以色列生活居住, 也说明了阿拉伯裔以色列人的国家归属感的显著提升。 调查问题e: 感觉到以色列国籍的重要性时, 在老一代 阿拉伯裔以色列人有 $75 \%$ 的老一代受访者认为该项目 是比较重要的, 而在新一代阿拉伯裔以色列人中这一 比例降到了 $52 \%$, 另外有 $31 \%$ 的新一代阿拉伯裔以色列 人认为感受到以色列国籍不是很重要。值得注意的是, 对于问题f: 拥有以色列祖先的重要性时, 无论是老一 代还是新一代阿拉伯裔以色列人绝大多数受访者都认 为拥有以色列祖先是不重要的, 这也说明阿拉伯裔以 色列人即使生活在以色列境内, 仍然保持着对自己的 阿拉伯祖先的强烈的认同感。

\section{4. 结论}

本文基于聚类分析对阿拉伯裔以色列人的国家归 属感进行了定量分析, 首先, 从年龄结构上对阿拉伯 裔进行划分, 新一代以色列阿拉伯人年龄在 30 岁左右, 有着良好的教育背景, 而老一代阿拉伯人年龄在平均 值在49.15岁受教育程度一般, 其平均受教育年限不到 10 年。其次分析了以色列裔阿拉伯人国家归属感情况, 结果显示: 阿拉伯裔以色列人的国家归属感总体状况 良好, 但新老两代阿拉伯裔以色列人的国家认同程度 存在明显差异。新一代阿拉伯裔以色列人的国家认同 明显高于老一代阿拉伯裔以色列人, 这表明以色列国 家认同建设取得了一定成效。但鉴于阿拉伯裔以色列 人身份的特殊性, 这种国家认同将来还会随着以色列 内部条件和外部环境的变化而改变, 从而影响以色列 的国家安全和社会稳定, 乃至影响整个中东地区的和 平与发展。

\section{REFERENCES}

[1] Shangtao Gao.A Study of Arab-Israeli Relations from the Perspective of Power Constructivism [M]. Beijng:World Affairs Press,2018

[2] Sarbu C, Nascu-Briciu R D, Kot-Wasik A, et al. Classification and fingerprinting of kiwi and pomelo fruits by multivariate analysis of chromatographic and spectroscopic data[J]. Food Chemistry, 2012, 130(4):994-1002.

[3] http://www.issp.org/menu-top/home/

[4] Xiaoyang Chen.Semantic Similarity Calculation of Short Text Based on Deep Learning[D]. Beijing: Beijing University of Technology,2015.

[5] RODRIGUEZ P, BAUTISTA M A, GONZALEZ $\mathrm{J}$, et al. Beyond one- hot encoding : lower dimensional target embedding[J]. Image and vision computing, 2018,75:21-31.

[6] Polykovskiy, D. and Novikov, A., Bayesian Methods for Machine Learning[J]. Coursera and National Research University Higher School of Economics, 2018.

[7] The Arab Population in Israel[J]. Israel Central Bureau of Statistics, Retrieved 9 July 2016.

[8] Jacob M. Landau. The Arab minority in Israel, 1967-1991: political aspects [M]. London: Oxford University Press, 1993.

[9] Wenjia Liu.A Study on The National Identity of Arab Israelis [D]. Beijing: Beijing Language and Culture University, 2019 